\title{
Towards Utility-based Resource Management in Heterogeneous Wireless Networks
}

\author{
Ilias Tsompanidis \\ Computer Science \\ Department \\ University College Cork, \\ Western Road, \\ Cork, Ireland \\ it1@cs.ucc.ie
}

\author{
Ahmed H. Zahran \\ Electronics and Electrical \\ Communications Department \\ Cairo University, \\ Gamaet El Qahera St., \\ Giza, Egypt \\ azahran@eece.cu.edu.eg
}

\author{
Cormac J. Sreenan \\ Computer Science \\ Department \\ University College Cork, \\ Western Road, \\ Cork, Ireland \\ cjs@cs.ucc.ie
}

\begin{abstract}
This work considers the network selection and the bandwidth assignment problems in the context of heterogeneous wireless networks operated by a single service provider. The current commercial and research practices on resource management are presented, and a novel utility-based approach supporting multiple client classes is introduced. A bandwidth sharing policy and a "controlled unfairness" scheme is achieved by combining distinct priority classes with logarithmic utility functions that variably grade the bandwidth allocated to clients.

To demonstrate the possibilities of this approach an optimisation problem is formulated and its solution utilityoptimally allocates bandwidth and distributes clients to base stations, modelling a network-side resource management system. The centralised operation allows for network-wide provision of comparable service levels to clients of the same class. The optimal solution is compared to several heuristic methods. Simulations showcase the behaviour of the system, which successfully differentiates clients and utilises all available resources.
\end{abstract}

\section{Categories and Subject Descriptors}

C.2.3 [Computer-Communication Networks]: Network Operations - network management, public networks; G.1.6 [Numerical Analysis]: Optimization-constrained optimization; J.4 [Social And Behavioral Sciences]: Economics

\section{General Terms}

Management, Design

\section{Keywords}

Heterogeneous Wireless Network, Priority class, Network Selection, Resource Management

Permission to make digital or hard copies of all or part of this work for personal or classroom use is granted without fee provided that copies are not made or distributed for profit or commercial advantage and that copies bear this notice and the full citation on the first page. To copy otherwise, to republish, to post on servers or to redistribute to lists, requires prior specific permission and/or a fee.

MobiArch'12, August 22, 2012, Istanbul, Turkey.

Copyright 2012 ACM 978-1-4503-1526-5/12/08 ...\$15.00.

\section{INTRODUCTION}

With the gradual deployment of $4 \mathrm{G}$ in parallel to existing $3 \mathrm{G}$ services, wireless service providers $(W S P \mathrm{~s})$ find themselves operating a number of different but spatially overlapping technologies and networks. At the same time, customers use more powerful mobile devices and demand better service, with reliable, high-speed connections, while consuming increasingly more multimedia content.

Global mobile data traffic is forecasted to increase 18-fold between 2011 and 2016, while the adoption of $4 \mathrm{G}$ networks will not be wide enough to accommodate this amount of traffic [1]. The unprecedented amount of traffic is urging WSPs to take unilaterally-decided measures to reduce traffic load, such as the abolition of unlimited data service plans and throttling for "heavy" users [4]. It is becoming obvious that WSPs are not prepared for this amount of traffic and that a mutually acceptable bandwidth management scheme is needed.

WSPs have not taken advantage of the increasing prevalence of multi-interface mobile devices. The management of resources is still limited to each access network technology, and WSPs have no means of directing client devices to selectively use a specific network. The unification of the management of access networks can be an important asset to WSPs, allowing them to spread clients over all available access networks, maximise capacity usage and achieve balanced utilisation.

In this paper, we present a new framework for network selection and resource allocation in a heterogeneous network operated by a single WSP. The framework considers users of multiple classes by defining different utility-based functions for each class, while the utility function definition describes the relation between users of different classes. This work discusses the optimal user differentiation and the full utilisation of resources the framework achieves, as well as the initial work on sub-optimal heuristic-based approximation methods.

The remainder of the paper is organised as follows. The next Section presents the current practices of WSPs. Section 3 provides insight on the use of utility functions as a means of quantifying the importance of allocated bandwidth to clients. The proposed framework that controls the allocation of network resources is defined in Section 4 and results are then presented in Section 5. Related work is reviewed in Section 6 and Section 7 gives a closing perspective on this ongoing research. 


\section{BACKGROUND}

WSPs have experimented with various types of service plans, such as "Pay per MB", "Flat-rate for unlimited data", and "Flat-rate for a specific data allowance". The data allowance model has proved to be a commercial success with customers, hence most WSPs offer a selection of tiered data allowance plans, letting end-users decide the amount of data they expect to consume over a time period and choose a fitting data plan. Previous research work, such as [13], is often based on the assumption that a customer signs agreements with multiple providers and chooses the one optimising the cost, QoS or bandwidth. However, due to the "always-on" nature of the modern Internet access paradigm, this practice can lead to unpredictable billing, whereas customers usually prefer having prior knowledge of service charges, and generally opt to subscribe to a flat-rate service plan with a single WSP [7]. Further discrepancies between real practices and research-driven enhancements are found by looking into how operators confront the network selection, bandwidth allocation, and client prioritisation problems.

The network selection problem is usually split into two distinct sub-problems, namely selecting a Radio Access Technology $(R A T)$ and selecting a Base Station $(B S)$. In this paper the term BS is used to denote any data-capable attachment point for mobile devices, such as cells or WiFi APs. With spatially overlapping $3 \mathrm{G}$ and $4 \mathrm{G}$ deployments, a vertical load balancing algorithm would be expected. Unfortunately, WSPs have little control on RAT selection, and they provide no incentives for clients to select a specific technology. In fact, most users have their devices configured to automatically select the "best" RAT, e.g. in a commonly found $2 \mathrm{G}-3 \mathrm{G}-\mathrm{WiFi}$ coexistence scenario, this policy selects the $3 \mathrm{G}$ network for voice calls and the WiFi network for data transmission.

Depending on the RAT implementations used by a WSP, horizontal load balancing algorithms can be employed, assisting the assignment of clients to BSs. Cell size optimisations, such as cell-breathing [17], are not uncommon, albeit usually applicable to intra-domain operations and limited by vendor-specific protocols.

Demanding users have forced WSPs to start considering the prioritisation and bandwidth allocation problems. Unfortunately, the measures taken by WSPs cause significant discontent. Users responsible for an excessive amount of traffic are punished via throttling or banned from the high capacity networks, effectively limiting service down to GPRS or EDGE levels. Throttling is the prevailing method for rate-limiting, even though deprioritisation can be much friendlier for end-users [19]. The main difference is that throttling constantly limits the throughput of specific clients, whereas deprioritisation characterises the traffic associated with these clients as low priority and limits its rate under congestion, but still allows high throughput when the traffic load of the network is low.

\section{CLASS-BASED UTILITY FUNCTIONS}

This work considers the problems of service differentiation and resource sharing in a single-WSP heterogeneous wireless network environment. We introduce the use of priority classes as an extension of WSP-provided service plans. For example, a WSP could decide to support three service plans, and offer them as: a) Gold user $(\boldsymbol{G C})$, the high priority class for users that demand the highest level of service, b) Silver user ( $S C$ ), the medium priority class for normal users, c) Bronze user $(B C)$, the low priority class for low-end users, or "punishment class" for users abusing the service.

It can be expected that the pricing of each service plan will reflect the corresponding user priority. Each user preselects a service plan that suits his needs, and agrees to a differentiating service, i.e. agrees to be entitled to a level of service in regard to all other users of the network. This level of service is controlled by the utility function each class is assigned. Please note that differentiation is not applied perflow, but on a per-client basis. The assignment of clients to priority classes is at least medium-term, i.e. it is not expected to change more than a couple of times in a day. In fact, the class of a client is assumed to change only if the user exceeds a data usage limit or manually updates the service plan.

Utility functions have been extensively used in Economics for investment and consumption modelling. Fig. 1a shows the three types of risk behaviour. Concave functions, such as logarithms, are considered to model a risk-averse behaviour, while linear and convex functions respectively represent riskneutral and risk-seeking behaviour [3, 8].

In the context of bandwidth management, a risk-averse user appreciates higher throughput rates, but demands a basic level of service, whereas a risk-seeking user is only satisfied with a high throughput rate. Risk-averse behaviour is linked to best-effort, elastic traffic [10]. In this work, the utility of a logarithmic function is a measure of importance of bandwidth $(f(x))$ to bandwidth $(x)$ per client. An example of a utility function, used throughout this paper, is:

$$
f_{P}(x)=P \ln \left(\frac{e-1}{P} x+1\right)
$$

where $P$ represents a tuning parameter to change the curvature of the utility function as shown in Fig. 1b. In this work, we consider different $P$ values of $0.6,0.3$, and 0.1 to respectively represent users belonging to $\mathrm{GC}, \mathrm{SC}$, and $\mathrm{BC}$. For such representation, the sum of utility for clients sharing a single BS is maximised for one optimal sharing ratio.

To further elaborate, consider three clients belonging to distinct classes and sharing a BS. The optimal bandwidth shares that maximise the utility would be $60 \%$ of the capacity for the GC client, $30 \%$ for the SC client, and $10 \%$ for the $\mathrm{BC}$ client. Fig. 1c shows the total utility and its single maximum of two clients of classes GC and SC, and bandwidth B1 and B2, when sharing a BS of capacity 1 bandwidth unit $(B u)$. The concavity of Eq. 1 ensures a single maximum utility for any number of clients sharing a single BS.

The per-client computation of utility alleviates the unfairness a per-class approach would impose, as clients, regardless of their number, would share a set amount of bandwidth for each class. The proposed functional form also provides a network-wide tendency to max-min fairness among clients of the same class [6].

\section{OPTIMAL RESOURCE MANAGEMENT}

In this section, we formulate the utility-based bandwidth allocation and BS assignment optimization framework. The objective is to maximize the sum of user utilities. Table 1 lists all the optimisation parameters used in Eq. 2. The operating scenario assumes a number of partially overlapping 


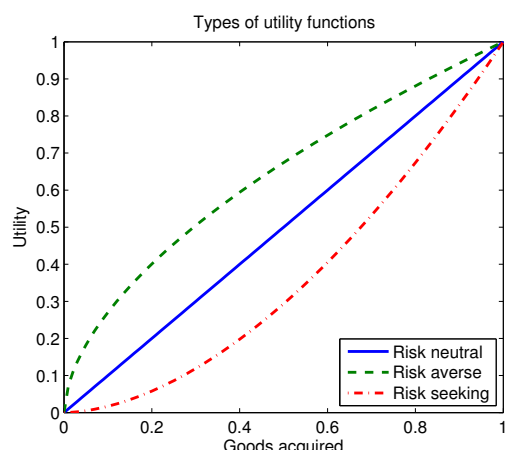

(a) Utility functions of risk-averse, riskneutral and risk-seeking behaviour.

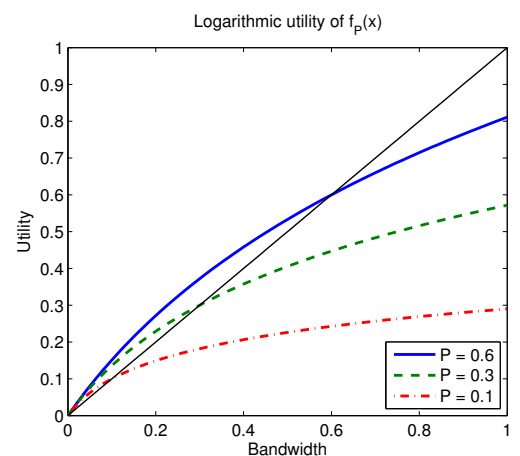

(b) Example of utility functions for 3 classes. $\mathrm{Y}$ axis is the utility (utils) and $\mathrm{X}$ axis is the allocated bandwidth $(\mathrm{Bu})$.

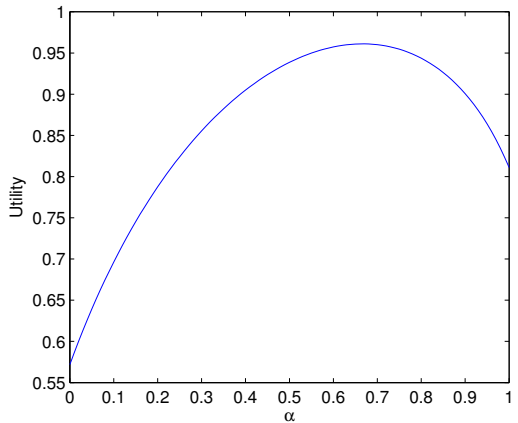

(c) Total utility of two clients belonging to classes $\mathrm{GC}$ and $\mathrm{SC}$, sharing a BS, with $B_{1}=\alpha, B_{2}=1-\alpha$.

Figure 1: Attributes of utility functions.

heterogeneous BSs, nomadic users requesting connections at the beginning of their sessions, and that a number of parameters are known to the optimising entity.

Maximise:

$$
U=\sum_{i} f_{P_{i}}\left(B_{i}\right)
$$

subject to:

$$
\begin{aligned}
& A N_{i, s}=\left\{\begin{aligned}
& 1, \text { if } i \text { assigned } \\
& \text { to } s \in\left(L_{i}\right) \\
& 0, \text { otherwise }
\end{aligned}\right. \\
& \sum_{s} A N_{i, s} \leq 1, \forall i \\
& \sum_{i} B_{i} A N_{i, s} \leq C_{s}, \forall s \\
& B_{i} A N_{i, s} \leq E M_{i, s}, \forall s, i \\
& B_{i} \leq E D_{i}, \forall i \\
& B_{i} \geq T_{P_{i}}, \forall i
\end{aligned}
$$

- Eq. $2 \mathrm{~b}$ and 2c mandate that each client $i$ associates to only one visible BS $s$.

- Eq. 2d limits the sum of allocated bandwidth of clients associated to a single BS s to its capacity.

- Eq. 2e indicates that user $i$ allocated bandwidth on BS $s$ is upper-bounded by the maximum achievable throughput on their corresponding link. Additionally, Eq. $2 \mathrm{f}$ indicates that user $i$ allocated bandwidth is also upper-bounded by its demand.

- Eq. $2 \mathrm{~g}$ imposes a lower bound for the allocated bandwidth for each user. Typically, this bound may be a common value for users belonging to the same class.

Eq. 2 describes a constrained, non-convex, non-linear problem. As an analytic approach is challenging and outside the scope of this paper, we produce the optimal solution by utilising a SQP solver starting from multiple starting points.

Typically, the network-side entity performing this optimisation should have a complete view of the network and be able to assess the parameters described in Table 1. Hence, a control messaging protocol, e.g. 802.21, can be used to
Table 1: Index of terms

Table 1: Index of terms
\begin{tabular}{|c|c|}
\hline Term & Symbol \\
\hline Client & $i$ \\
\hline Base Station (BS) & $s$ \\
\hline Priority class of Client $i$ & $P_{i}$ \\
\hline Bandwidth allocated for Client $i$ & $B_{i}$ \\
\hline List of visible BS to Client $i$ & $L_{i}$ \\
\hline Maximum capacity of BS $s$ & $C_{s}$ \\
\hline Maximum achievable throughput & \\
estimation of Client $i$ associated to BS $s$ & $E M_{i, s}$ \\
\hline $\begin{array}{c}\text { Estimated Service Rate demand } \\
\text { of Client } i \text { (BS-independent) }\end{array}$ & $E D_{i}$ \\
\hline Minimum Bandwidth threshold for $P_{i}$ & $T_{P_{i}}$ \\
\hline Boolean indicator of client $i$ & \\
association to BS $s$ & $A N_{i, s}$ \\
\hline
\end{tabular}

transfer the information gathered by the client, such as $L_{i}$, or available at the client, such as the $E D_{i}$, to the central entity responsible for resource allocation and bandwidth assignment. This entity solves the optimization problem and sends the assigned BSs and bandwidth shares information back to the client.

\section{PERFORMANCE ANALYSIS}

The performance of the proposed Utility-based Resource Management (URM) is evaluated using MATLAB. First we present the evaluation setup, followed by the obtained results.

\subsection{Simulation Setup}

In our evaluation, we consider three overlapping generic BSs, named Fast, Normal, and Slow. These BSs could correspond to different technologies in next generation systems. We also implement a number of client data rates depending on the connection quality, with the supported rates varying between maximum and minimum values with a regular step as shown in Table 2.

The expected demand $(E D)$ of the clients is generated using a uniform distribution. The minimum demand is 0 bandwidth units $(B u)$ and the maximum demand is $40 \mathrm{Bu}$. It is also assumed that each client can communicate with the simulated BSs at a randomly selected data rate correspond- 
Table 2: BS Data Rates (Bu)

\begin{tabular}{|c|c|c|c|}
\hline BS & Min & Max & Step \\
\hline Fast & 30 & 300 & 30 \\
\hline Normal & 10 & 100 & 10 \\
\hline Slow & 1.5 & 15 & 1.5 \\
\hline
\end{tabular}

ing to the connection quality. The number of the clients is chosen such that the network load is 1.5 the network capacity. Additionally, each client is assigned a random priority class according to a uniform probability marginal function.

We compare URM with different heuristics for network selection and bandwidth sharing strategies. For network selection, we consider:

1. RSS-Equivalent (RSS): A client-side strategy in which the client connects to the BS with the best estimated achievable throughput.

2. Maximum Available Bandwidth (MAB): A network side strategy in which the client is connected to the BS with the most available bandwidth.

On the occasion of insufficient available bandwidth for the traffic demand at one of the BSs, bandwidth sharing adopts one of the following strategies:

1. Fair Sharing (FS): Each client is allocated an equal share of the resources of the BS (water-filling). Note that the reallocation of resources takes place with the user arrivals and departures.

2. Proportional Degradation (PD): Each client is allocated a bandwidth proportional to its expected demand with respect to the total traffic demand at this BS.

3. Weighted Sharing (CS): Similar to FS, however, the bandwidth shares ratio for each client depends on the class of the client.

These algorithms are compared on the basis of the average total utility, total throughput, fairness index, and denied bandwidth. For fairness, we consider the Jain index, estimated as:

$$
F I=\frac{\left(\sum \frac{B_{i}}{E D_{i}}\right)^{2}}{n \sum\left(\frac{B_{i}}{E D_{i}}\right)^{2}}
$$

\subsection{Evaluation Results}

For the shown results, $E M_{i, s}$ is assumed the maximum data rate of $s$. It is worth noting that randomly assigned $E M$ produces similar qualitative results. Figures $2 \mathrm{a}-2 \mathrm{~d}$ respectively show the per-class average of the total utility, total throughput, fairness index, and denied bandwidth for different combinations of network selection and bandwidth sharing strategies. As expected, URM strikes the optimal utility for the main service classes (Gold and Silver) without a significant reduction in the utility of lower priority class as shown in Fig. 2a. A similar observation is clear for the attained average throughput per class. Additionally, URM realises a fair service for $\mathrm{GC}$ users and relatively acceptable fairness for both SC and BC. URM also successfully prioritises GC users and achieves the least average denied bandwidth per user as shown in Fig. 2c. The main drawback of URM is scalability due to the mixed nature of the optimisation problem. Practically, URM can be used as a benchmark for other simpler heuristics.

Currently, RSS-based strategies represent a natural dominant behaviour for BS selection. As shown in the figures, RSS-based strategies, independent of the resource sharing mechanism, deviate significantly from the optimal behaviour of URM. Note that clients adopting RSS-based strategies in the simulated scenario tend to select the fast BS as a default service point. This selection would drop the total system capacity to the fast BS capacity ignoring the other two BSs. Additionally, it is also worth noting that the current bandwidth sharing behaviour of WSPs mostly resembles PD.

In contrast to RSS-based schemes, MAB allocates clients to all BSs. As shown in Fig. 2b MAB-FS and MAB-PD deviate from the optimal target performance realised by URM. On the other hand, the performance of MAB-CS closely matches the optimal behaviour leading to a near optimal performance.

To further understand the behaviour of URM and MABCS, Fig. 3a focuses on the clients for which the allocated bandwidth does not satisfy their demands, showcasing the bandwidth sharing facilities of the two algorithms. While URM is more stable, providing a similar service level to clients belonging in the same class, MAB-CS is more unpredictable, spreading allocated bandwidth values over a wider range. Fig. 3b shows the average the standard deviation of the throughput per class for URM and MAB-CS for the same clients, further validating the previous remarks.

To this end, it is worth pointing out that the assigned weights per client in our evaluation match the utility function priorities for different classes; i.e. 0.6, 0.3, 0.1 for GC, SC, and BC respectively. Such utility-inspired weighing strategy motivates utility-based MAB-CS as a practical, network-based, combined BS selection and bandwidth sharing strategy. Further tests will help ensure the applicability and consistency of such heuristic methods under a wider variety of network conditions, as well as identify their behaviour with increasing client mobility.

\section{RELATED WORK}

Received signal strength (RSS) is the most common metric used for network selection. RSS provides significant information about the quality of the connection, even though the shortcomings of relying only on RSS-based metrics are known and have been presented by many researchers. Improvements have been made with handoff hysteresis and RSS prediction algorithms (e.g. [5]), however, signal strength is only one of the parameters for optimal network selection.

Pérez-Romero et al. [14] address the issue of RAT selection by introducing a sophisticated metric, namely the fittingness factor, reflecting the degree of adequacy of a RAT to a given user, in terms of available QoS level and technical terminal capabilities. A fuzzy logic control and MultiCriteria Decision Making (MCDM) system for network selection is developed by Alkhawlani and Hussein [2], considering reliability, security, battery power and price as criteria.

SUTIL [16] is a distributed network selection solution, based on utility function and integer linear programming, taking into account bandwidth, packet loss, delay and energy information. Unfortunately, the need to establish base 


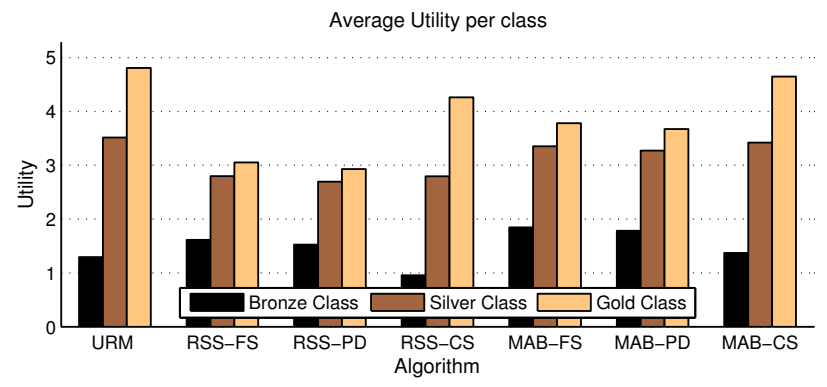

(a) Total utility per class.

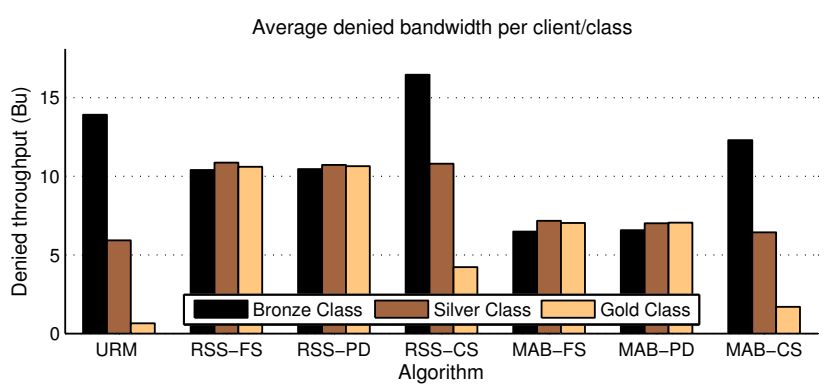

(c) Denied bandwidth per client/class.

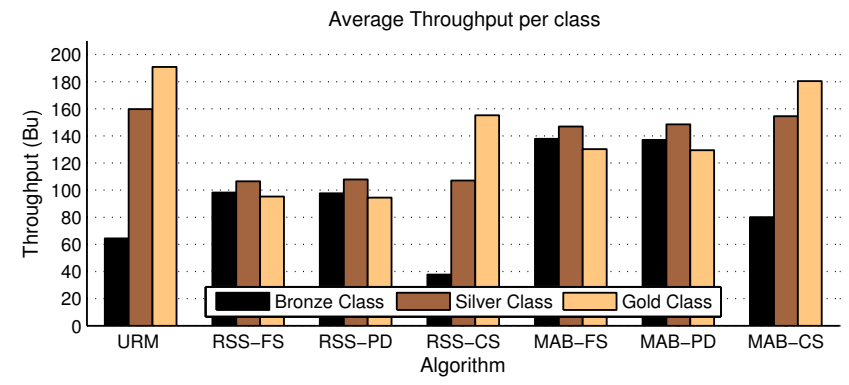

(b) Total throughput per class. URM prioritises higher class traffic to achieve higher utility.

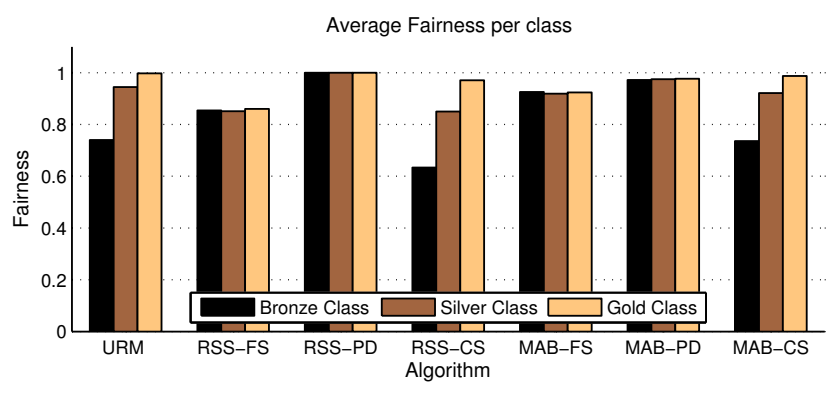

(d) Fairness per class.

Figure 2: Average statistics over 100 snapshots.

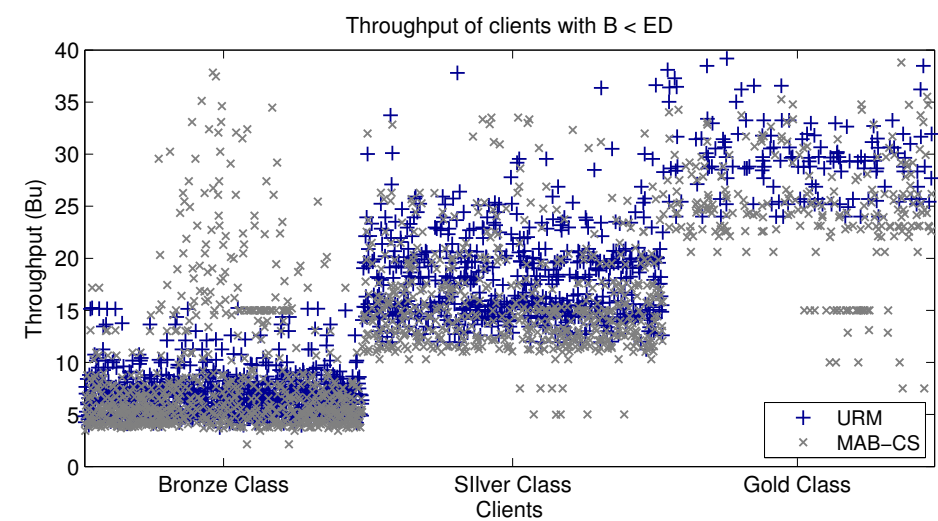

(a) Throughput per client.

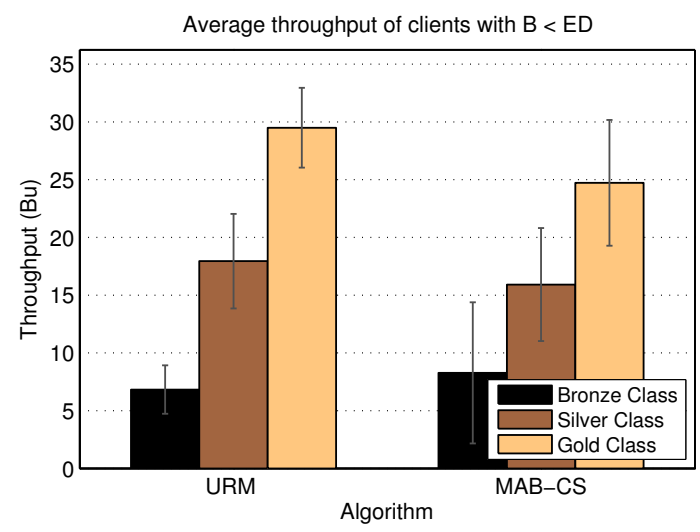

(b) Average and standard deviation of throughput per class.

Figure 3: Throughput of clients with $B_{i}<E D_{i}$.

requirements for every service used in the network and the assumption that the total network bandwidth is enough to sustain all traffic are unrealistic.

The big-picture view that WSPs have of their network can prove to be of great significance when considering the assignment of clients to BSs. The centralised Common Radio Resource Management (CRRM) scheme [15], is a move towards a tightly integrated network management environment. Clients are assigned to BSs optimising the overall network performance, but not necessarily the performance of the client. An admission and handover protocol in the context of CRRM is discussed by Jin et al. [11], employing a load-based marginal cost function for optimal BS selection.

Young et al. [18] describe the network selection problem from the perspective of a Virtual Network Operator, match- ing clients to access networks. They employ degradable QoS profiles, assigning clients to operators offering the best QoS, and forcing QoS degradation on a per-flow basis if needed. However, the logistics of assigning every flow type to a class and a degradation profile discourage a real system implementation of similar approaches.

An interesting centralised resource management algorithm is proposed by Jia et al. [9], where a bits/Hz ratio minimisation problem is presented, effectively optimising spectrum usage. The optimisation problem approach is similar to our work, though it focuses on spectrum allocation, whereas we propose a resource sharing system that supports multiple priority classes.

Utility maximisation approaches have been used for resource allocation in wireless networks $[10,12]$, offering ana- 
lytical methods to solve the resource management problem in a single BS. We are extending this work by presenting an optimisation framework for a heterogeneous wireless environment and multiple client classes.

Our work defines a bandwidth sharing scheme between user classes and optimally assigns clients to BSs by combining the use of priority classes and network-wide user differentiation with a WSP-operated resource management system.

\section{CONCLUSION AND FUTURE WORK}

This paper proposes a utility-based resource management framework that differentiates user classes by means of a risk-averse utility function. The optimisation problem for this complicated system is identified and formulated, while the analytic study of the solution will be part of our future work. This preliminary work displays the benefits of such an approach, particularly in the context of a heterogeneous wireless network, and attempts to identify simpler heuristicbased algorithms that approximate the optimal solution.

A number of issues have to be resolved for a practical application of this research, such as scalability. The centralised approach, used for simplicity in this paper, and the optimisation complexity, make real-time operation for large number of devices challenging. Future work includes a decentralised approach that reduces the problem set by considering smaller geographical regions and improvements on the optimisation speed by finding relaxation approaches and utilising guiding heuristics.

This paper also indicated an algorithm that approximates the optimal solution, not affected by scalability issues. The consistency of this sub-optimal approach needs to be verified in a wider set of environments.

While the evaluated system provides optimal solutions for snapshots of the network, more work needs to be done towards stability under client mobility and traffic variability. Aspects that will be investigated include the tolerance of sub-optimal solutions and the applicability of traffic forecasting.

\section{ACKNOWLEDGMENTS}

This work is supported by Science Foundation Ireland (SFI) under Research Grant 10RFP/CMS2952.

\section{REFERENCES}

[1] Cisco Visual Networking Index: Global Mobile Data Traffic Forecast Update, 2011-2016. White Paper, February 2012.

[2] M. M. Alkhawlani and A. A. Hussein. Intelligent Radio Network Delection for Next Generation Networks. In Proc. Int. Conf. Informatics and Systems, pages 1-7, March 2010.

[3] K. Arrow. Essays in the Theory of Risk Bearing. North-Holland, Amsterdam, 1976.

[4] AT\&T. Data Usage Information \& FAQs, March 2012. http://www.att.com/datainfo.

[5] B.-J. Chang and J.-F. Chen. Cross-Layer-Based Adaptive Vertical Handoff With Predictive RSS in Heterogeneous Wireless Networks. Vehicular Technology, 57(6):3679-3692, November 2008.

[6] A. Coluccia, A. D'Alconzo, and F. Ricciato. On the optimality of maxmin fairness in resource allocation.
Annals of Telecommunications, 67(1-2):15-26, January-February 2012.

[7] T. J. Gerpott. Biased choice of a mobile telephony tariff type: Exploring usage boundary perceptions as a cognitive cause in choosing between a use-based or a flat rate plan. Telematics and Informatics, 26(2):167-179, May 2009.

[8] D. Hernández-Hernández and A. Schied. Robust maximization of consumption with logarithmic utility. In Proc. American Control Conf., pages 1120-1123, July 2007.

[9] H. Jia, Z. Zhang, P. Cheng, H.-H. Chen, and S. Li. Study on Network Selection for Next-Generation Heterogeneous Wireless Networks. In Proc. Int. Symp. Personal, Indoor and Mobile Radio Communications, pages 1-5, September 2006.

[10] Z. Jiang, Y. Ge, and Y. Li. Max-Utility Wireless Resource Management for Best-Effort Traffic. Wireless Communications, 4(1):100-111, January 2005.

[11] F. Jin, H.-A. Choi, J.-H. Kim, J. Sohn, and H. I. Choi. Common radio resource management for access selection in multi-access networks. In Proc. Radio and Wireless, pages 643-646, January 2008.

[12] W.-H. Kuo and W. Liao. Utility-Based Resource Allocation in Wireless Networks. Wireless Communications, 6(10):3600-3606, October 2007.

[13] O. Ormond, G.-M. Muntean, and J. Murphy. Economic Model for Cost Effective Network Selection Strategy in Service Oriented Heterogeneous Wireless Network Environment. In Proc. Symp. Network Operations and Management, April 2006.

[14] J. Pérez-Romero, O. Sallent, and R. Agusti. A Novel Metric for Context-Aware RAT Selection in Wireless Multi-Access Systems. In Proc. Int. Conf. Communications, pages 5622-5627, June 2007.

[15] J. Pérez-Romero, O. Sallent, R. Agusti, P. Karlsson, A. Barbaresi, L. Wang, F. Casadevall, M. Dohler, H. González, and F. Cabral-Pinto. Common radio resource management: functional models and implementation requirements. In Proc. Int. Symp. Personal, Indoor and Mobile Radio Communications, pages 2067-2071, September 2005.

[16] L. Pirmez, J. C. Carvalho, Jr., F. C. Delicato, F. Protti, L. F. R. C. Carmo, P. F. Pires, and M. Pirmez. SUTIL - Network selection based on utility function and integer linear programming. Computer Networks, 54:2117-2136, September 2010.

[17] K. L. Thng, B. S. Yeo, and Y. H. Chew. Performance Study on the Effects of Cell-Breathing in WCDMA. In Proc. Int. Symp. Wireless Communication Systems, pages 44-49, September 2005.

[18] X. Yang, J. Bigham, and L. Cuthbert. Resource management for service providers in heterogeneous wireless networks. In Proc. Conf. Wireless Communications and Networking, pages 1305-1310, March 2005.

[19] H. Zhou, K. Sparks, N. Gopalakrishnan, P. Monogioudis, F. Dominique, P. Busschbach, and J. Seymour. Deprioritization of heavy users in wireless networks. Communications, 49(10):110-117, October 2011. 